\title{
On the three ball theorem for solutions of the Helmholtz equation
}

\author{
Stine Marie Berge ${ }^{1} \cdot$ Eugenia Malinnikova $^{1,2}$
}

Published online: 13 April 2021

(c) The Author(s) 2021

\begin{abstract}
Let $u_{k}$ be a solution of the Helmholtz equation with the wave number $k, \Delta u_{k}+k^{2} u_{k}=0$, on (a small ball in) either $\mathbb{R}^{n}, \mathbb{S}^{n}$, or $\mathbb{\mathbb { A } ^ { n }}$. For a fixed point $p$, we define $M_{u_{k}}(r)=\max _{d(x, p) \leq r}\left|u_{k}(x)\right|$. The following three ball inequality

$M_{u_{k}}(2 r) \leq C(k, r, \alpha) M_{u_{k}}(r)^{\alpha} M_{u_{k}}(4 r)^{1-\alpha}$

is well known, it holds for some $\alpha \in(0,1)$ and $C(k, r, \alpha)>0$ independent of $u_{k}$. We show that the constant $C(k, r, \alpha)$ grows exponentially in $k$ (when $r$ is fixed and small). We also compare our result with the increased stability for solutions of the Cauchy problem for the Helmholtz equation on Riemannian manifolds.
\end{abstract}

Keywords Helmholtz equation · Almgren's frequency function · quantitative uniqueness

Mathematics Subject Classification 47A75 - 35J05 · 58J05

\section{Introduction}

In the present work, we study constants in the three ball inequality for solutions of the Helmholtz equation. We begin by recalling Hadamard's celebrated three circle theorem. Let $f$ be a holomorphic function in the disk $\mathbb{D}_{R}=\{z \in \mathbb{C}:|z|<R\}$. Then its maximum function

$M_{f}(r)=\max _{|z| \leq r}|f(z)|$

satisfies the convexity condition

$M_{f}\left(r_{0}^{\alpha} r_{1}^{1-\alpha}\right) \leq M_{f}\left(r_{0}\right)^{\alpha} M_{f}\left(r_{1}\right)^{1-\alpha}$,

for any $r_{0}, r_{1}<R$ and $\alpha \in(0,1)$. The proof of (1.1) is based on the fact that $\log |f|$ is a subharmonic function. Note that

Stine Marie Berge

stine.m.berge@ntnu.no

Eugenia Malinnikova

eugeniam@stanford.edu

1 Department of Mathematical Sciences, Norwegian University of Science and Technology, 7491 Trondheim, Norway

2 Department of Mathematics, Stanford University, Stanford, CA 94305, USA by the maximum principle (1.1) also holds when the maximum is taken over circles.

Surprisingly, Hadamard's theorem generalizes to other classes of functions, such as solutions of second order elliptic equations and their gradients. We refer the reader to the article [11] of Landis and to the survey [1]. Three spheres theorems for the gradients of harmonic functions and, more generally, harmonic differential forms can be found in [15]. The three ball theorem for solutions of the Helmholtz equation on Riemannian manifolds were studied in [16]. This has various applications, for example it was one of the tools used to estimate the Hausdorff measure of the nodal sets of Laplace eigenfunctions, see $[12,13]$.

We consider the Helmholtz equation

$\Delta_{M} u_{k}+k^{2} u_{k}=0$

on a domain $D$ in a Riemannian manifold $(M, \mathbf{g})$. For $D=M$ and $M$ being a closed manifold without boundary, solutions of (1.2) are $L^{2}$-eigenfunctions of the Laplacian. One of the important facts for analysis on closed manifolds is the existence of an orthonormal basis for $L^{2}(M)$ consisting of eigenfunctions of the Laplacian. The classical example is the Fourier basis on the circle $\mathbb{S}^{1}$. Such an orthonormal basis can be used to solve the heat, wave, and Schrödinger equations on closed manifolds, under certain conditions. 
We study properties of functions that satisfy the Helmholtz equation on some geodesic ball in the manifold. Fix a point $p \in M$ and denote by $B(p, r)$ the geodesic ball of radius $r$ centered at $p$. Then for a function $u$ we define

$M_{u}(r)=\max _{x \in B(p, r)}|u(x)|$.

The following doubling inequality holds for Laplace eigenfunctions on a closed manifold

$M_{u_{k}}(2 r) \leq C_{1} \mathrm{e}^{C_{2} k} M_{u_{k}}(r)$,

where $C_{1}$ and $C_{2}$ are constants only depending on the Riemannian manifold $(M, \mathbf{g})$. Inequality (1.3) was first shown by Donnelly and Fefferman [5]. Later Mangoubi [16, Theorem 3.2] gave a new proof by showing the stronger local inequality

$M_{u_{k}}(3 r) \leq C_{3} \mathrm{e}^{C_{4} k r} M_{u_{k}}(2 r)^{\alpha} M_{u_{k}}(8 r)^{1-\alpha}$,

for small $r$, some fixed $\alpha \in(0,1)$, and constants $C_{3}$ and $C_{4}$ only depending on the curvature. Further results on the propagation of smallness for eigenfunctions were obtained in [14]. In this article, we show that (1.4) is sharp in the following sense: The coefficient $C_{3} \mathrm{e}^{C_{4} k r}$ in (1.4) cannot be replaced by a function growing subexponentially in $k r$ as $k$ grows. This is done by constructing special families of solutions of the Helmholtz equation on Euclidean spaces, hyperbolic spaces, and the standard spheres.

We also compare (1.4) with the increased stability for solutions of the Cauchy problem for the Helmholtz equation studied in $[3,8,10]$. Roughly speaking, the idea is that one can estimate the solution in the interior of some convex domain from an a priori bound and an estimate of the Cauchy data on some part of the boundary. Moreover, the estimate does not depend on $k$. For solutions of the Helmholtz equation in a geodesic ball $B(p, R)$ we prove for $r<R_{1}<R$ that

$\int_{B(p, r)} u_{k}^{2} \mathrm{dvol} \leq C\left(r, R_{1}\right) \int_{B\left(p, R_{1}\right) \backslash B(p, r)} u_{k}^{2} \mathrm{dvol}$,

and call (1.5) the reverse three ball inequality. A more general result can be found in [2, Sect. 1.3], where delicate questions regarding localization of solutions of the Schrödinger equation are considered. We deduce (1.5) from a similar estimate for the $H^{1}$ norms where the constant does not depend on $k$. The $H^{1}$ estimate is proved by a Carleman-type inequality, that can be found in $[3,9]$.

The structure of the paper is as follows. We prove the sharpness of the three ball inequality (1.4) in Sect. 2 . In Sect. 2.1 we present the argument for the Euclidean space, while the arguments for the hyperbolic space and the sphere are given in Sect. 2.2. We prove inequality (1.5) in Sect. 3. Finally, we give a simple estimate for the location of the first positive zero of the Bessel functions, and collect some comparison theorems for solutions of the Sturm-Liouville equations in "Appendix".

\section{The three ball inequality}

\subsection{Bessel functions and the Helmholtz equation in $\mathbb{R}^{n}$}

Let $J_{l}$ denote the Bessel function of the first kind. We have collected some facts about the Bessel functions in "Appendix A". If $Y_{m}$ is an eigenfunction of the Laplace operator on the sphere $\mathbb{S}^{n-1}$ with eigenvalue $m(m+n-2)$ then

$u_{k}(r, \theta)=r^{1-n / 2} J_{m+n / 2-1}(k r) Y_{m}(\theta)$

solves the Helmholtz equation (1.2). Moreover, any solution of (1.2) in $\mathbb{R}^{n}$ (or in the unit ball) can be decomposed into a series of such solutions.

In order to study the constant in the three ball inequality (1.4) that involves the maximum function, we analyze the behavior of the Bessel functions. From now on we assume that $n=2$ for simplicity. Our results can be easily extended to all dimensions $n \geq 2$.

Lemma 2.1 Let $0<\gamma<\delta<1$ and set $\beta=\sqrt{1-\delta^{2}}$. Then there exists a constant $C$, only depending on $\gamma$ and $\delta$, such that for any positive number $m$ we have

$J_{m}(\gamma m)<C\left(\frac{\gamma}{\delta}\right)^{\beta m} J_{m}(\delta m)$.

Proof The strategy is to apply the Sturm comparison theorem, see Theorem B.2. We apply the theorem to the Bessel function $J_{m}$ solving the Bessel equation

$\left(x J_{m}^{\prime}(x)\right)^{\prime}+\frac{x^{2}-m^{2}}{x} J_{m}(x)=0$,

and a solution of the Euler equation

$\left(x y^{\prime}(x)\right)^{\prime}+\frac{\left(\delta^{2}-1\right) m^{2}}{x} y(x)=0$.

Let $y$ be the solution of (2.2) satisfying the initial conditions $y(\gamma m)=J_{m}(\gamma m) \quad$ and $\quad y^{\prime}(\gamma m)=J_{m}^{\prime}(\gamma m)$.

We know that $J_{m}$ is positive and increasing on $[0, m]$. The latter can be verified using the second derivative test and inserting the argument of the first maximum of $J_{m}$ into the equation 
$x^{2} J_{m}^{\prime \prime}(x)+x J_{m}^{\prime}(x)+\left(x^{2}-m^{2}\right) J_{m}(x)=0$.

Moreover, notice that for $x \in[\gamma m, \delta m]$ we have

$x^{2}-m^{2} \leq\left(\delta^{2}-1\right) m^{2}$.

Hence all the conditions in the comparison theorem are satisfied and we conclude that $y(x) \leq J_{m}(x)$ on $[\gamma m, \delta m]$.

Any solution of the Euler equation (2.2) is on the form

$y(x)=c_{1} x^{m \beta}+c_{2} x^{-m \beta}$.

Using that

$J_{m}(\gamma m)=y(\gamma m)>0 \quad$ and $\quad J_{m}^{\prime}(\gamma m)=y^{\prime}(\gamma m)>0$,

we conclude that $c_{1}>0$ and $\left|c_{2}\right|<c_{1} \gamma^{2 m \beta} m^{2 m \beta}$. Thus

$J_{m}(\gamma m)=c_{1}(\gamma m)^{m \beta}+c_{2}(\gamma m)^{-m \beta}<2 c_{1}(\gamma m)^{m} \beta$

and

$y(\delta m)>q c_{1}(\delta m)^{m \beta}$,

where $q=q(\gamma, \delta)>0$. It follows that

$$
\begin{aligned}
J_{m}(\gamma m) & <2 c_{1}(\gamma m)^{m \beta}<\frac{2}{q}\left(\frac{\gamma}{\delta}\right)^{m \beta} y(\delta m) \\
& <\frac{2}{q}\left(\frac{\gamma}{\delta}\right)^{m \beta} J_{m}(\delta m) .
\end{aligned}
$$

We can now prove the main result of this section.

Theorem 2.2 Assume that there is an $\alpha \in(0,1)$ and a constant $C(k, r, \alpha)$ such that for any solution $u_{k}$ of the Helmholtz equation (1.2) the following three ball inequality holds

$M_{u_{k}}(2 r) \leq C(k, r, \alpha) M_{u_{k}}(r)^{\alpha} M_{u_{k}}(4 r)^{1-\alpha}$.

Then $C(k, r, \alpha)$ grows at least exponentially in $k r$. More precisely, $C(k, r, \alpha) \geq c \mathrm{e}^{d \alpha k r}$, where $c$ and $d$ are absolute constants.

Proof Consider solutions of the Helmholtz equation on the form

$u_{k}(r, \theta)=J_{m}(k r) \sin (m \theta)$.

The maximal function then simplifies to

$M_{u_{k}}(r)=\max _{0 \leq x \leq k r}\left|J_{m}(x)\right|$.

We now use the fact that for $m>0$ the maximum of $J_{m}(x)$ is attained in the interval $(m, m(1+\varepsilon(m)))$, where $\varepsilon(m) \rightarrow 0$ as $m \rightarrow \infty$. This is a well known result on the asymptotic of the first zero of the Bessel functions, for the convenience of the reader we include a simple proof in "Appendix A". We choose $m_{0}$ such that $\varepsilon(m) \leq 1 / 3$ when $m \geq m_{0}$. Assume first that

$k r>m_{1}=\max \left\{4,2 m_{0} / 3\right\}$.

Then given $r$ we can find $m \geq m_{0}$ such that

$6 k r / 5<m<3 k r / 2$.

This implies $k r<5 m / 6$ and $2 k r>4 / 3 m$. Then $M_{u_{k}}(4 r)=M_{u_{k}}(2 r)$ and we can reduce (2.3) to

$\left(\frac{M_{u_{k}}(2 r)}{M_{u_{k}}(r)}\right)^{\alpha} \leq C(k, r, \alpha)$.

Set $\gamma=5 / 6$ and $\delta=\frac{1+\gamma}{2}=\frac{11}{12}$. Applying Lemma 2.1 together with

$M_{u_{k}}(2 r)>J_{m}(m)>J_{m}(\delta m)$

and $M_{u_{k}}(r)<J_{m}(\gamma m)$ we conclude that

$C(k, r, \alpha) \geq\left(\frac{M_{u_{k}}(2 r)}{M_{u_{k}}(r)}\right)^{\alpha} \geq c \mathrm{e}^{\alpha d m}$,

for some positive constant $d$ that can be computed. Finally, since $m>6 k r / 5$ we get the required estimate when $r>k^{-1} m_{1}$.

Now for $r \leq k^{-1} m_{1}$ we consider the solution $u_{k}(r, \theta)=J_{0}(k r)$. Then $M_{u_{k}}(r)=J_{0}(0)$ since

$J_{0}(0)=\max _{x \geq 0}\left|J_{0}(x)\right|$,

and we conclude that $C(k, r, \alpha) \geq 1$ for any $r>0$. Choosing $c<\mathrm{e}^{-\alpha d m_{1}}$ we have for all $r>0$ that

$C(k, r, \alpha) \geq c \mathrm{e}^{\alpha d k r}$.

\subsection{Solutions of the Helmholtz equation on the sphere and hyperbolic space}

In this section we repeat the argument of the sharpness of the three ball inequality on the hyperbolic space and sphere. We show in particular that assumptions on the sign of the curvature do not lead to better behavior of the constant in the three ball inequality. Again, we use the spherical symmetry of the spaces and separation of variables to construct a solution of (1.2) that is the product of a radial and a spherical factor. 
On the sphere the radial part is given by Legendre polynomials. For the hyperbolic space the radial part is also explicitly known, see [4, p. 4222 Eq. (2.26)]. Once again, in our argument we only use the differential equation for the radial part.

We define

$\sin _{K}(r)= \begin{cases}\frac{\sin (\sqrt{K} r)}{\sqrt{K}}, & \text { when } K>0 \\ r, & \text { when } K=0 . \\ \frac{\sinh (\sqrt{-K} r)}{\sqrt{-K}}, & \text { when } K<0\end{cases}$

Furthermore, we use the associated functions $\cos _{K}(r)=\left(\sin _{K}(r)\right)^{\prime}, \cot _{K}(r)=\frac{\cos _{K}(r)}{\sin _{K}(r)}$, and $\tan _{K}(r)=\frac{1}{\cot _{K}(r)}$. Then the Laplacian of a simply connected $n$-dimensional Riemannian manifold $(M, \mathbf{g})$ with constant sectional curvature $K$ is given in polar coordinates by

$\Delta_{M}=\frac{\mathrm{d}^{2}}{\mathrm{~d} r^{2}}+(n-1) \cot _{K}(r) \frac{\mathrm{d}}{\mathrm{d} r}+\frac{1}{\sin _{K}^{2}(r)} \Delta_{\mathbb{S}^{n-1}}$.

In this section, we work in two dimension. Assume that $u_{k}(r, \theta)=R(r) \Theta(\theta)$ is a solution of the Helmholtz equation. Then $R(r)$ satisfies the equation

$$
\begin{gathered}
\sin _{K}^{2}(r)\left(\frac{R^{\prime \prime}(r)+\cot _{K}(r) R^{\prime}(r)}{R(r)}+k^{2}\right) \\
=-\frac{\Delta_{\mathbb{S}^{1}} \Theta(\theta)}{\Theta(\theta)}=m^{2} .
\end{gathered}
$$

Let $\kappa=K / k^{2}$ and let $L_{\kappa, m}(\rho)$ be the solution of the differential equation

$$
\begin{aligned}
& \sin _{\kappa}^{2}(\rho) L_{\kappa, m}^{\prime \prime}(\rho)+\sin _{\kappa}(\rho) \cos _{\kappa}(\rho) L_{\kappa, m}^{\prime}(\rho) \\
& +\left(\sin _{\kappa}^{2}(\rho)-m^{2}\right) L_{\kappa, m}(\rho)=0,
\end{aligned}
$$

where $L_{\kappa, m}$ is well-defined at $\rho=0$ and positive on some interval $(0, \varepsilon)$. Then for $m>0$ we have $L_{\kappa, m}(0)=0$. Note that when $K=0$ this equation becomes the Bessel equation. Setting $R(r)=L_{\kappa, m}(k r)$ we get a solution to (2.5). Then (2.6) can be rewritten in the Sturm-Liouville form as

$\left(\sin _{\kappa}(\rho) L_{\kappa, m}^{\prime}(\rho)\right)^{\prime}+\frac{\sin _{\kappa}^{2}(\rho)-m^{2}}{\sin _{\kappa}(\rho)} L_{\kappa, m}(\rho)=0$.

We begin by estimating the maximum point of $L_{\kappa, m}$ from below. Let

$R_{\kappa}= \begin{cases}\infty, & \kappa \leq 0 \\ \frac{\pi}{2 \sqrt{\kappa}}, & \kappa>0\end{cases}$

Note that for $\rho \leq R_{\kappa}$ we have that $\sin _{\kappa}(\rho)$ is increasing, or equivalently that $\cos _{\kappa}(\rho) \geq 0$.
Proposition 2.3 Let $0<\rho_{1}^{*}<\rho_{2}^{*}<\cdots<R_{\kappa}$ be the points where $L_{\kappa_{\mathrm{p}} m}$ attains local maximums and minimums before $R_{\kappa}$. Then $\left[L_{\kappa, m}\left(\rho_{i}^{*}\right) \mid\right.$ is a decreasing sequence in $i$. Moreover, the first local maximum $\rho_{1}^{*}$ satisfies $\rho_{1}^{*} \geq \sin _{\kappa}^{-1}(m)$.

Proof At $\rho_{1}^{*}$ we have $L_{\kappa, m}^{\prime}\left(\rho_{1}^{*}\right)=0$ and (2.7) implies that $\sin _{\kappa}^{2}\left(\rho_{1}^{*}\right) L_{\kappa, m}^{\prime \prime}\left(\rho_{1}^{*}\right)+\left(\sin _{\kappa}^{2}\left(\rho_{1}^{*}\right)-m^{2}\right) L_{\kappa, m}\left(\rho_{1}^{*}\right)=0$.

By the second derivative test it is not possible to have a maximum before $\sin _{\kappa}^{-1}(m)$, implying the lower bound for the first local extremum.

The remaining part of the proposition follows from Sonin-Pólya oscillation theorem, see Theorem B.3. The conditions in the oscillation theorem are satisfied on the interval $\left(\sin _{\kappa}^{-1}(m), R_{\kappa}\right)$ since $\sin _{\kappa}(\rho)>0$,

$\left(\sin _{\kappa}^{2}(\rho)-m^{2}\right) / \sin _{\kappa}(\rho) \neq 0$,

and

$\left(\sin _{\kappa}(\rho) \frac{\sin _{\kappa}^{2}(\rho)-m^{2}}{\sin _{\kappa}(\rho)}\right)^{\prime}=2 \cos _{\kappa}(\rho) \sin _{\kappa}(\rho)>0$.

Thus the sequence $\left|L_{\kappa, m}\left(\rho_{i}^{*}\right)\right|$ is decreasing.

Remark 2.4 For $m=0$, by analyzing the differential equation (2.6), we see that $L_{\kappa, 0}^{\prime}(0)=0$. Then the proof of Proposition 2.3 implies that $L_{\kappa, 0}(\rho)$ satisfies $L_{\kappa, 0}(0) \geq\left|L_{\kappa, 0}(\rho)\right|$ for $\rho>0$.

Now our aim is to prove an analog of Lemma 2.1. The next four results show how we can control the ratio of two values of $L_{\kappa, m}$.

Lemma 2.5 Let $\rho_{2} \in\left(0, R_{\kappa}\right)$ and $\delta \in(0,1)$ satisfy the inequality $\sin _{\kappa}\left(\rho_{2}\right) \leq \delta m$. Then for $\rho_{1}<\rho_{2}$ and $\beta=\sqrt{1-\delta^{2}}$ we have the bound

$\frac{L_{\kappa, m}\left(\rho_{2}\right)}{L_{\kappa, m}\left(\rho_{1}\right)} \geq \frac{1}{2}\left[\left(\frac{\tan _{\kappa}\left(\rho_{2} / 2\right)}{\tan _{\kappa}\left(\rho_{1} / 2\right)}\right)^{\beta m}-\left(\frac{\tan _{\kappa}\left(\rho_{2} / 2\right)}{\tan _{\kappa}\left(\rho_{1} / 2\right)}\right)^{-\beta m}\right]$.

Proof We compare the function $L_{\kappa, m}$ to a solution of the equation

$\left(\sin _{\kappa}(\rho) y^{\prime}(\rho)\right)^{\prime}+\frac{m^{2}\left(\delta^{2}-1\right)}{\sin _{\kappa}(\rho)} y(\rho)=0$.

By the assumption we have

$\sin _{\kappa}^{2}(\rho)-m^{2} \leq\left(\delta^{2}-1\right) m^{2}=-\beta^{2} m^{2}$

on the interval $\left[\rho_{1}, \rho_{2}\right]$. Let $y$ be the solution to $(2.8)$ that satisfies the initial conditions 
$y\left(\rho_{1}\right)=L_{\kappa, m}\left(\rho_{1}\right)$ and $y^{\prime}\left(\rho_{1}\right)=L_{\kappa, m}^{\prime}\left(\rho_{1}\right)$.

Then the comparison theorem implies that $L_{\kappa, m}\left(\rho_{2}\right)>y\left(\rho_{2}\right)$.

The explicit solution to (2.8) is given by

$y(\rho)=c_{1} \tan _{\kappa}^{\beta m}(\rho / 2)+c_{2} \tan _{\kappa}^{-\beta m}(\rho / 2)$.

The first maximum $\rho_{1}^{*}$ of $L_{\kappa, m}$ satisfies $\sin _{\kappa}\left(\rho_{1}^{*}\right) \geq m$ implying that $\rho_{2}<\rho_{1}^{*}$. Therefore $L_{\kappa, m}\left(\rho_{1}\right)>0$ and $L_{\kappa, m}^{\prime}\left(\rho_{1}\right)>0$. Thus we have the inequality

$-c_{1} \tan _{\kappa}^{2 \beta m}\left(\rho_{1} / 2\right)<c_{2}<c_{1} \tan _{\kappa}^{2 \beta m}\left(\rho_{1} / 2\right)$,

since $\left(\tan _{\kappa}(\rho / 2)\right)^{\prime}>0$. We conclude that $c_{1}>0$ and similarly to Lemma 2.1 we get

$$
\begin{aligned}
& L_{\kappa, m}\left(\rho_{2}\right)>y\left(\rho_{2}\right) \\
& \quad>c_{1}\left(\tan _{\kappa}^{\beta m}\left(\rho_{2} / 2\right)-\tan _{\kappa}^{2 \beta m}\left(\rho_{1} / 2\right) \tan _{\kappa}^{-\beta m}\left(\rho_{2} / 2\right)\right) .
\end{aligned}
$$

The estimate of $c_{2}$ from below implies that

$L_{\kappa, m}\left(\rho_{1}\right)=y\left(\rho_{1}\right)<2 c_{1} \tan _{\kappa}^{\beta m}\left(\rho_{1} / 2\right)$.

Combining the last two inequalities gives the result.

Corollary 2.6 Suppose that $K>0$ and that $\rho_{1}<\rho_{2}<\min \left\{R_{\kappa}, m \delta\right\}$ for some $\delta \in(0,1)$. For $\beta=\sqrt{1-\delta^{2}}$ we have the estimate

$\frac{L_{\kappa, m}\left(\rho_{2}\right)}{L_{\kappa, m}\left(\rho_{1}\right)} \geq \frac{1}{2}\left[\left(\frac{\rho_{2}}{\rho_{1}}\right)^{\beta m}-\left(\frac{\rho_{2}}{\rho_{1}}\right)^{-\beta m}\right]$.

Proof We note that $\sin _{\kappa}\left(\rho_{2}\right)<\rho_{2}<m \delta$. Applying Lemma 2.5 and using the elementary inequality $b \tan x \geq \tan b x$ for $b \in(0,1)$, the result follows since

$\frac{\tan _{\kappa}\left(\rho_{2} / 2\right)}{\tan _{\kappa}\left(\rho_{1} / 2\right)}=\frac{\tan \left(\sqrt{\kappa} \rho_{2} / 2\right)}{\tan \left(\sqrt{\kappa} \rho_{1} / 2\right)} \geq \frac{\rho_{2}}{\rho_{1}}$.

\section{Corollary 2.7 Let $K<0$ and suppose that}

$\rho_{1}<\rho_{2}<\min \left\{R_{|\kappa|}, 2 m \delta / 3\right\}$

for some $\delta \in(0,1)$. Then for $\beta=\sqrt{1-\delta^{2}}$ and $A=\sin _{\kappa}\left(\rho_{2}\right) / \rho_{2}$ we have

$\frac{L_{\kappa, m}\left(\rho_{2}\right)}{L_{\kappa, m}\left(\rho_{1}\right)} \geq \frac{1}{2}\left[\left(\frac{\rho_{2}}{A \rho_{1}}\right)^{\beta m}-\left(\frac{\rho_{2}}{A \rho_{1}}\right)^{-\beta m}\right]$.

Proof Since $\sqrt{|\kappa|} \rho<\pi / 2$ and sinh is convex we have

$\sin _{\kappa}\left(\rho_{2}\right) \leq 2 \rho_{2} \sinh (\pi / 2) / \pi<3 \rho_{2} / 2<m \delta$.

Applying Lemma 2.5 together with $(\log (\tanh x))^{\prime} \geq \frac{\rho_{2} \sqrt{-\kappa}}{\sinh \left(\rho_{2} \sqrt{-\kappa}\right) x} \quad$ for $x<\rho_{2} \sqrt{-\kappa} / 2$

gives (2.10), since

$\frac{\tan _{\kappa}\left(\rho_{2} / 2\right)}{\tan _{\kappa}\left(\rho_{1} / 2\right)}=\frac{\tanh \left(\sqrt{|\kappa|} \rho_{2} / 2\right)}{\tanh \left(\sqrt{|\kappa|} \rho_{1} / 2\right)} \geq \frac{\rho_{2}}{A \rho_{1}}$.

We want to estimate the ratio of the values of $L_{\kappa, m}$ at two points $\rho_{2}>\rho_{1}>\sin _{\kappa}^{-1}(m)$. In contrast with the Bessel functions, we do not locate the maximum precisely.

Lemma 2.8 Suppose that $0<\rho_{1}<R_{|\kappa|}$ and $\sin _{\kappa}\left(\rho_{1}\right)>\xi m$, where $\xi>1$. There is an absolute constant $C>0$ such that

$\frac{\max _{\rho}\left|L_{\kappa, m}(\rho)\right|}{\max _{\rho \leq \rho_{1}}\left|L_{\kappa, m}(\rho)\right|} \leq 1+\frac{C}{(\xi-1) m}$.

Proof Let $\rho_{1}^{*}$ be the first local maximum of $L_{\kappa, m}$. By Proposition 2.3 if $\rho_{1}>\rho_{1}^{*}$ then the left-hand side of $(2.11)$ is one and the statement becomes trivial.

The rest of the proof relies on the comparison of $L_{\kappa, m}$ and a solution of the equation

$\left(\sin _{\kappa}(\rho) y^{\prime}\right)^{\prime}+\frac{m^{2}\left(\xi^{2}-1\right)}{\sin _{\kappa}(\rho)} y=0$

on the interval $\left(\rho_{1}, \infty\right)$. Solutions to (2.12) are of the form

$y(\rho)=c_{1} \cos \left(\gamma \log \left(\tan _{\kappa}(\rho / 2)\right)\right)+c_{2} \sin \left(\gamma \log \left(\tan _{\kappa}(\rho / 2)\right)\right)$,

where $\gamma^{2}=\xi^{2}-1$. Let $d=\gamma \log \left(\tan _{\kappa}\left(\rho_{1} / 2\right)\right)$ and choose a solution $y$ of (2.12) on the form

$y(\rho)=C_{1} \cos \left(\gamma \log \left(\tan _{\kappa}(\rho / 2)\right)-d\right)$

$$
+C_{2} \sin \left(\gamma \log \left(\tan _{\kappa}(\rho / 2)\right)-d\right),
$$

with initial data $y\left(\rho_{1}\right)=L_{\kappa, m}\left(\rho_{1}\right)$ and $y^{\prime}\left(\rho_{1}\right)=L_{\kappa, m}^{\prime}\left(\rho_{1}\right)$. This gives the values

$C_{1}=L_{\kappa, m}\left(\rho_{1}\right), \quad C_{2}=\frac{L_{\kappa, m}^{\prime}\left(\rho_{1}\right) \sin _{\kappa}\left(\rho_{1}\right)}{\gamma}$.

Applying the comparison theorem, we get

$$
\begin{aligned}
L_{\kappa, m}\left(\rho_{2}\right) \leq C_{1} & \cos \left(\gamma \log \left(\tan _{\kappa}\left(\rho_{2} / 2\right)\right)-d\right) \\
& +C_{2} \sin \left(\gamma \log \left(\tan _{\kappa}\left(\rho_{2} / 2\right)\right)-d\right) .
\end{aligned}
$$

Since sin and $\cos$ are bounded by 1 , we estimate $C_{2} / L_{\kappa, m}\left(\rho_{1}\right)$ from above to prove (2.11). In order to estimate $C_{2}$, we see that the assumption $\rho_{1}<\rho_{1}^{*}$ implies $L_{\kappa, m}(\rho)>0$ and $L_{\kappa, m}^{\prime}(\rho)>0$ on the interval $\left(0, \rho_{1}\right)$. Equation (2.7) shows that 
$L_{\kappa, m}^{\prime \prime}(\rho)<0$ when $\rho \in\left(\rho_{0}, \rho_{1}\right)$, where $\rho_{0}=\sin _{\kappa}^{-1}(m)$. Then the Taylor formula gives

$L_{\kappa, m}\left(\rho_{0}\right)-L_{\kappa, m}\left(\rho_{1}\right)+\left(\rho_{1}-\rho_{0}\right) L_{\kappa, m}^{\prime}\left(\rho_{1}\right)<0$.

Consequently,

$L_{\kappa, m}^{\prime}\left(\rho_{1}\right)<\frac{L_{\kappa, m}\left(\rho_{1}\right)-L_{\kappa, m}\left(\rho_{0}\right)}{\rho_{1}-\rho_{0}}<\frac{L_{\kappa, m}\left(\rho_{1}\right)}{\rho_{1}-\rho_{0}}$.

For $K>0$ the inequality

$x_{1}-x_{0}>\sin x_{1}-\sin x_{0} \quad$ when $\quad x_{1}>x_{0}$

implies that $\rho_{1}-\rho_{0}>(\xi-1) m$. For $K<0$, we note that

$x_{1}-c \sinh x_{1}>x_{0}-c \sinh x_{0}$ when $x_{1}>x_{0}$,

if $\cosh x_{1}<c^{-1}$. Using the assumption $\rho_{1}<R_{|\kappa|}$, we conclude that $\rho_{1}-\rho_{0}>c(\xi-1) m$, where $c=(\cosh \pi / 2)^{-1}$. Finally, we obtain

$$
\begin{aligned}
\max _{\rho \geq \rho_{1}}\left|L_{\kappa, m}(\rho)\right| & \leq \sqrt{C_{1}^{2}+C_{2}^{2}} \\
& \leq L_{\kappa, m}\left(\rho_{1}\right)\left(1+\frac{C}{(\xi-1) m}\right) .
\end{aligned}
$$

Now we are ready to prove that the coefficient in the three ball theorem grows exponentially in $r k$ if we restrict ourselves to balls with sufficiently small radius $r$.

Theorem 2.9 Let $(M, \mathbf{g})$ be either a hyperbolic plane or a sphere and denote its curvature by $K$. Suppose that for some $\alpha \in(0,1)$ there exists a constant $C_{\alpha}(k, r, K)$ such that for any solution $u_{k}$ to the Helmholtz equation (1.2) the following inequality holds

$$
\begin{aligned}
& M_{u_{k}}(2 r) \leq C_{\alpha}(k, r, K) M_{u_{k}}(r)^{\alpha} M_{u_{k}}(4 r)^{1-\alpha}, \\
& 0<r<\frac{\pi}{8 \sqrt{|K|}} .
\end{aligned}
$$

\section{Then}

$C_{\alpha}(k, r, K) \geq c_{1}^{\alpha} \mathrm{e}^{c_{2} \alpha k r}$,

where $c_{1}$ and $c_{2}$ only depend on $K$.

Proof Consider the family of functions

$u_{k, m}(r, \theta)=L_{\kappa, m}(k r) \sin (m \theta)$, where $m$ is a non-negative integer. By construction, $u_{k, m}$ solves the Helmholtz equation. Thus for any $m$ we have the inequality

$C_{\alpha}(k, r, K) \geq\left(\frac{M_{m}(2 k r)}{M_{m}(k r)}\right)^{\alpha}\left(\frac{M_{m}(2 k r)}{M_{m}(4 k r)}\right)^{1-\alpha}$,

where

$M_{m}(\rho)=\max _{x \leq \rho}\left|L_{\kappa, m}(x)\right|$.

Note that choosing $m=0$ gives $C_{\alpha}(k, r, K) \geq 1$ by Remark 2.4. Thus if we assume that $k r<C_{1}$ for some constant $C_{1}$, we may choose $c_{2}$ and $c_{1}$ small enough such that the inequality holds.

Assume first that $K<0$ so that $(M, \mathbf{g})$ is the hyperbolic plane. If $k r>C_{1}$ we choose a positive integer $m$ such that $10 m<18 k r<11 m$. We apply (2.10) with $\rho_{1}=k r$ and $\rho_{2}=\mu k r<2 k r$, where $\mu=19 / 17$. Then $\rho_{2}<2 / 3 m \delta$ with $\delta<1$. We obtain

$\frac{M_{m}(2 k r)}{M_{m}(k r)} \geq \frac{L_{\kappa, m}\left(\rho_{2}\right)}{L_{\kappa, m}\left(\rho_{1}\right)} \geq \frac{1}{2}\left[\left(\frac{\rho_{2}}{A \rho_{1}}\right)^{\beta m}-\left(\frac{\rho_{2}}{A \rho_{1}}\right)^{-\beta m}\right]$,

where $\beta=\sqrt{1-\delta^{2}}$ and

$$
\begin{aligned}
A & =\sin _{\kappa}\left(\rho_{2}\right) / \rho_{2}<\sin _{\kappa}(2 k r) /(2 k r) \\
& <4 \sinh (\pi / 4) / \pi<10 / 9 .
\end{aligned}
$$

Therefore $q=\mu / A>1$ is an absolute constant and we have $\frac{M_{m}(2 k r)}{M_{m}(k r)} \geq \frac{1}{2}\left(q^{\beta m}-q^{-\beta m}\right)$.

Thus there are $c_{1}>0$ and $c_{2}>0$ such that

$M_{m}(2 k r) \geq c_{1} \exp \left(c_{2} m\right) M_{m}(k r)$.

On the other hand, we have $2 k r>\xi m$ for $\xi=10 / 9$. Applying (2.11) we get

$\frac{M_{m}(4 r)}{M_{m}(2 r)}=\frac{\max _{\rho \leq 4 k r}\left|L_{\kappa, m}(\rho)\right|}{\max _{\rho \leq 2 k r}\left|L_{\kappa, m}(\rho)\right|} \leq 1+C / m \leq C_{0}$,

where $C_{0}$ is an absolute constant. Note also that $m \gtrsim k r$. Then (2.13) follows for negative curvature.

Assume now that $K>0$ so that $(M, \mathbf{g})$ is a sphere. If $k r>C_{1}$ we choose $m$ to be a positive integer such that $10 \mathrm{~m}<12 \mathrm{kr}<11 \mathrm{~m}$. We first let $\rho_{1}=k r$ and $\rho_{2}=13 \mathrm{kr} / 12$ and apply (2.9) with $\delta=143 / 144$. Thus (2.14) follows whenever $k r>C_{1}$. Using (2.11) with $\rho_{1}=2 k r$ we need to check that $2 \rho_{1}>\xi \pi m$ for some $\xi>1$. Note that 
$2 \rho_{1}=4 k r>10 / 3 m$ and choose $\xi<\frac{10}{3 \pi}$. Then (2.13) follows for positive curvature.

\section{The reverse three ball inequality}

The question of stability of the solution to the Cauchy problem for the Helmholtz equation and the dependence of the estimates on the wave number $k$ was studied by many authors, see e.g., $[3,8,10,19]$. We include a special case of the results adapted to the case of Riemannian manifolds to demonstrate the difference between the usual three ball theorem and the reverse one.

Let $(M, \mathbf{g})$ be a Riemannian manifold with sectional curvature satisfying

$\kappa \mathbf{g}(X, X) \leq \sec (X, X) \leq K \mathbf{g}(X, X)$.

We denote by $\operatorname{grad}_{M}$ and $\Delta_{M}$ the gradient and Laplace operators on functions on $M$. Let $B$ be a geodesic ball with diameter strictly less than the injectivity radius of $(M, \mathbf{g})$. Additionally, in the case that $K>0$ we assume that the diameter of $B$ is strictly less than $\frac{\pi}{2 \sqrt{K}}$.

Theorem 3.1 Let $u_{k}$ solve the Helmholtz equation $\Delta_{M} u_{k}+k^{2} u_{k}=0$ in $B=B(p, R)$ and let $r<R_{1}<R$. There exists $C=C\left(r, R_{1}\right)$ such that

$\int_{B(p, r)} u_{k}^{2} \mathrm{dvol} \leq C\left(r, R_{1}\right) \int_{B\left(p, R_{1}\right) \backslash B(p, r)} u_{k}^{2} \mathrm{dvol}$,

The result is very closed to a particular case of the result in [3], we sketch the proof for the convenience of the reader.

We say that a function $\phi: B \rightarrow \mathbb{R}$ is strictly convex if its Hessian is positive definite. We choose a point $x$ such that $x \notin B(p, R)$ but $R+\operatorname{dist}(x, p)$ is strictly less than the injectivity radius and than $\frac{\pi}{2 \sqrt{K}}$ for the case $K>0$, and consider $\phi(y)=\operatorname{dist}(x, y)^{2}$. This function is smooth on $B$ since the metric on the Riemannian manifold is assumed to be smooth and $x \notin B$ while $B$ is contained in the ball of the injectivity radius around $x$. Moreover, Hess $(\phi)$ is (uniformly) positive definite on $B$ and $\phi$ has no critical points, see [18, Theorem 6.4.8] and the preceding discussions. By repeating the computations of [3, Lemma 1], where it is also pointed out that the result holds on Riemannian manifolds, we obtain the following point-wise inequality. Let $w \in C^{2}(B)$ and let $v=\mathrm{e}^{t \phi} w$, then

$$
\begin{aligned}
\mathrm{e}^{2 t \phi}\left(\Delta_{M} w+k^{2} w\right)^{2} \geq & 2 \operatorname{div}\left(b \operatorname{grad}_{M} v+a\right) \\
& +4 t\left\langle\operatorname{Hess}_{M}(\phi) \operatorname{grad}_{M} v, \operatorname{grad}_{M} v\right\rangle_{M} \\
& +4 t^{3}\left\langle\operatorname{Hess}_{M} \phi \operatorname{grad}_{M} \phi, \operatorname{grad}_{M} \phi\right\rangle_{M} v^{2} \\
& +t\left\langle\operatorname{grad}_{M} \Delta_{M} \phi, \operatorname{grad}_{M} v\right\rangle_{M} v,
\end{aligned}
$$

where $b=-t v \Delta_{M} \phi-2 t\left\langle\operatorname{grad}_{M} v, \operatorname{grad}_{M} \phi\right\rangle_{M}$ and

$a=t\left(\left|\operatorname{grad}_{M} v\right|_{M}^{2}-\left(k^{2}+t^{2}\left|\operatorname{grad}_{M} \phi\right|_{M}^{2}\right) v^{2}\right) \operatorname{grad}_{M} \phi$.

Lemma 3.2 Let $(M, \mathbf{g})$ and $B$ be as above. Then there exists a constant $c_{0}>0$ such that for any function $w \in C_{0}^{2}(B)$ and $k \geq 0$ the following inequality holds

$$
\int_{B}\left|\Delta_{M} w+k^{2} w\right|^{2} \mathrm{dvol} \geq c_{0} \int_{B}|w|^{2}+\left|\operatorname{grad}_{M} w\right|_{M}^{2} \text { dvol. }
$$

Proof We repeat the argument given in [3, Corollary 1]. Integrating the last inequality over a ball $B$ and taking into account that functions $a$ and $b$ have compact supports in $B$, we conclude that the divergence term disappears. For the next two terms, which contain the Hessian of $\phi$, we use the convexity inequality

$\left\langle\operatorname{Hess}_{M} \phi \operatorname{grad}_{M} f, \operatorname{grad}_{M} f\right\rangle_{M} \geq c_{\phi}\left|\operatorname{grad}_{M} f\right|_{M}^{2}$

and the computation

$\operatorname{grad}_{M} v=\mathrm{e}^{t \phi}\left(\operatorname{grad}_{M} w+t w \operatorname{grad}_{M} \phi\right)$.

Finally, the last term is estimated as

$\left|t\left\langle\operatorname{grad}_{M} \Delta_{M} \phi, \operatorname{grad}_{M} v\right\rangle_{M} v\right|$

$$
\leq \varepsilon t\left|\operatorname{grad}_{M} v\right|_{M}^{2}+\varepsilon^{-1} t\left|\operatorname{grad}_{M} \Delta_{M} \phi\right|_{M}^{2} v^{2} .
$$

Combining these inequalities, we get

$$
\begin{aligned}
& \int_{B}\left|\Delta_{M} w+k^{2} w\right|^{2} \mathrm{e}^{2 t \phi} \mathrm{dvol} \\
& \quad \geq c_{1} \int_{B}\left(t^{3}|w|^{2} \mathrm{e}^{2 t \phi}+t|\operatorname{grad} w|^{2} \mathrm{e}^{2 t \phi}\right) \mathrm{dvol},
\end{aligned}
$$

when $t>t_{0}$. The powerful feature of the last inequality is that $c_{1}$ and $t_{0}$ do not depend on $k$ (but depend on $\phi$ which we fix). Finally, we fix some $t>t_{0}$ and let $M=\max _{B} \mathrm{e}^{2 t \phi}$ and $m=\min _{B} \mathrm{e}^{2 t \phi}$. Then (3.2) holds with $c_{0}=c_{1} m \min \left\{t^{3}, t\right\} M^{-1}$. 
Suppose now that $u_{k}$ is a solution of the Helmholtz equation (1.2) in a ball $B(p, R)$ that satisfies the conditions in Theorem 3.1. We apply inequality (3.2) to $w=u_{k} \chi$, where $\chi \in C_{0}^{2}(B)$ is compactly supported on $B$ and equals to one on a smaller ball $B_{1} \subset \subset B$. This gives the inequality

$$
\begin{aligned}
& \int_{B_{1}}\left|u_{k}\right|^{2}+\left|\operatorname{grad} u_{k}\right|^{2} \mathrm{dvol} \\
& \quad \leq \frac{1}{C_{0}} \int_{B \backslash B_{1}}\left|u_{k} \Delta_{M} \chi+2 \operatorname{grad} u_{k} \cdot \operatorname{grad} \chi\right|^{2} \mathrm{dvol} .
\end{aligned}
$$

The last inequality implies that for any $r<R$ such that $B_{R}=B(x, R)$ and $B_{r}=B(x, r)$ are geodesic balls satisfying the conditions in Lemma 3.2, there is a constant $C_{2}(r, R)$ such that

$$
\begin{aligned}
& \int_{B_{r}}\left|u_{k}\right|^{2}+\left|\operatorname{grad} u_{k}\right|^{2} \mathrm{dvol} \\
& \quad \leq C_{2}(r, R) \int_{B_{R} \backslash B_{r}}\left|u_{k}\right|^{2}+\left|\operatorname{grad} u_{k}\right|^{2} \text { dvol. }
\end{aligned}
$$

Inequality (3.3) shows that if $u_{k}^{2}+\left|\operatorname{grad} u_{k}\right|^{2}$ is small on the annulus $B_{R} \backslash B_{r}$, then it is small on the whole ball $B_{R}$. For the Euclidean space an alternative proof can be obtained by decomposing a solution $u_{k}$ into series of products of Bessel functions and spherical harmonics. From this, one can deduce (3.3) from the Debye asymptotic of the Bessel functions.

To compare with the previous section and finish the proof Theorem 3.1, we can also use Caccioppoli's inequality to control the Sobolev norm of $u_{k}$ by its $L^{2}$-norm.

Lemma 3.3 (Caccioppoli's inequality) Let $\varepsilon>0$ and let $R=R(M)$ be small enough. Furthermore, let $\varepsilon<r<R-2 \varepsilon$. We denote

$$
\Omega=B(x, R) \backslash B(x, r),
$$

$\Omega_{+}=B(x, R+\varepsilon) \backslash B(x, r-\varepsilon), \quad$ and

$\Omega_{-}=B(x, R-\varepsilon) \backslash B(x, r+\varepsilon)$.

Assume that $u_{k} \in C^{2}(\Omega)$ and $\Delta_{M} u_{k}+k^{2} u_{k}=0$ in $\Omega_{+}$. Then there exists a constant $C=C(M)$ such that

$$
\begin{aligned}
& k^{2} \int_{\Omega_{-}} u_{k}^{2} \mathrm{dvol}-\frac{C}{\varepsilon^{2}} \int_{\Omega} u_{k}^{2} \mathrm{dvol} \\
& \quad \leq \int_{\Omega}\left|\operatorname{grad} u_{k}\right|^{2} \text { dvol } \\
& \quad \leq\left(k^{2}+\frac{C}{\varepsilon^{2}}\right) \int_{\Omega_{+}} u_{k}^{2} \mathrm{dvol} .
\end{aligned}
$$

Proof There exists a smooth function $\varphi_{+}$with compact support in $\Omega_{+}$that satisfy $\varphi_{+}=1$ on $\Omega$ and $\left|\operatorname{grad} \varphi_{+}\right| \leq \frac{C}{\varepsilon}$ and $\left|\Delta_{M} \varphi_{+}\right| \leq \frac{C}{\varepsilon^{2}}$. Then, using the divergence theorem, we have

$$
\begin{aligned}
k^{2} \int_{\Omega_{+}} \varphi_{+} u_{k}^{2} \mathrm{dvol}= & -\int_{\Omega_{+}} \varphi_{+} u_{k} \Delta_{M} u_{k} \mathrm{dvol} \\
= & \int_{\Omega_{+}}\left\langle\operatorname{grad} u_{k}, \operatorname{grad}\left(\varphi_{+} u_{k}\right)\right\rangle \mathrm{dvol} \\
= & \int_{\Omega_{+}} \varphi_{+}\left|\operatorname{grad} u_{k}\right|^{2} \mathrm{dvol} \\
& -\frac{1}{2} \int_{\Omega_{+}} u_{k}^{2} \Delta_{M} \varphi_{+} \mathrm{dvol} .
\end{aligned}
$$

Hence

$\int_{\Omega}\left|\operatorname{grad} u_{k}\right|^{2} \mathrm{dvol} \leq\left(k^{2}+C \varepsilon^{-2}\right) \int_{\Omega_{+}} u_{k}^{2} \mathrm{dvol}$.

On the other hand, choosing a similar function $\varphi_{-} \in C_{0}^{\infty}(\Omega)$ such that $\varphi_{-}=1$ on $\Omega_{-}$, we conclude that

$\int_{\Omega}\left|\operatorname{grad} u_{k}\right|^{2} \mathrm{dvol} \geq k^{2} \int_{\Omega_{-}} u_{k}^{2}-C \varepsilon^{-2} \int_{\Omega} u_{k}^{2} \mathrm{dvol}$.

Finally, we go back to the inequality (3.3), and apply the Caccioppoli inequality. Rename $R_{1}=R+\varepsilon$ and $r_{1}=r-\varepsilon$. This gives the following estimate of the $L^{2}$-norm of a solution to the Helmholtz equation by its $L^{2}$ norm on an annulus

$$
\begin{aligned}
k^{2} \int_{B_{r_{1}}} u_{k}^{2} \mathrm{dvol} \leq & \int_{B_{r}}\left|\operatorname{grad} u_{k}\right|^{2} \mathrm{dvol}+C \varepsilon^{-2} \int_{B_{r}} u_{k}^{2} \mathrm{dvol} \\
\leq & \left(k^{2}+C \varepsilon^{-2}\right) \int_{B_{R_{1}} \backslash B_{r_{1}}} u_{k}^{2} \mathrm{dvol} \\
& +C \varepsilon^{-2} \int_{B_{r_{1}}} u_{k}^{2} \mathrm{dvol},
\end{aligned}
$$

for any $r_{1}<R_{1}$. Then for $k>C \varepsilon^{-1}$ the inequality (1.5) follows. For $k<C \varepsilon^{-1}$, we use (3.3) and the Caccioppoli inequality again, to see that

$\int_{B_{r}}\left|u_{k}\right|^{2} \mathrm{dvol} \leq C(r, R)\left(k^{2}+C \varepsilon^{2}\right) \int_{B_{R_{1}} \backslash B_{r_{1}}}\left|u_{k}\right|^{2}$ dvol.

Thus, since $k<C \varepsilon^{-1}$, the inequality (1.5) follows also for that case. This conclude the proof of Theorem 3.1.

\section{Appendix A: The first positive zero of the Bessel function}

Let $l$ be a non-negative half-integer, and let $\Gamma$ denotes the gamma function. The Bessel function $J_{l}$ is a solution to the second order ODE

$\rho^{2} J_{l}^{\prime \prime}(\rho)+\rho J_{l}^{\prime}(\rho)+\left(\rho^{2}-l^{2}\right) J_{l}(\rho)=0$, 
which is bounded at the origin and normalized by the condition

$\lim _{\rho \rightarrow 0} \rho^{-l} J_{l}(\rho)=2^{-l} \Gamma(l+1)^{-1}$.

For alternative definitions and many useful asymptotic formulas for the Bessel functions we refer the reader to [17].

It is well known that the first positive zero of $J_{l}$, usually denoted by $j_{l}$, satisfies $j_{l} \asymp l+c l^{1 / 3}$ as $l \rightarrow \infty$. We already explained in the proof of Lemma 2.1 that $j_{l}>l$ and we give a simple proof of the inequality $j_{l} \leq l+c l^{1 / 3}$. This will be done by comparing the Bessel equation to the following equation

$\rho^{2} y_{l}^{\prime \prime}(\rho)+\rho y_{l}^{\prime}(\rho)+\left(a_{l}^{2} \rho^{2}-1 / 4\right) y_{l}(\rho)=0$

on the interval $\rho \in\left[l+l^{1 / 3},+\infty\right)$.

Suppose that $a_{l}<1$ satisfies

$\left(l+l^{1 / 3}\right)^{2}\left(1-a_{l}^{2}\right) \geq l^{2}-1 / 4$.

Then the Sturm-Picone comparison theorem, see Theorem B.1, implies that between any two zeros of $y_{l}$ there is a zero of $J_{l}$. It is easy to check that $y_{l}(\rho)=\rho^{-1 / 2} \cos \left(a_{l} \rho\right)$ solves (A.2) and has roots at $(\pi / 2+k \pi) / a_{l}$ for $k \in \mathbb{Z}$. We choose $a_{l}=l^{-1 / 3}$. Then (A.3) holds for $l$ large enough. Hence $J_{l}$ has a root on the interval $\left[l+l^{1 / 3}, l+l^{1 / 3}+\pi l^{1 / 3}\right]$ and $j_{l} \leq l+(\pi+1) l^{1 / 3}$.

\section{Appendix B: Comparison theorems for Sturm-Liouville equations}

Classical Sturm-Liouville theory is concerned with second order differential equations on the form

$\left(p(x) y^{\prime}(x)\right)^{\prime}+q(x) y(x)=0$ on $[a, b]$.

Special cases of Sturm-Liouville equations are the radial solutions to the Helmholtz equation, see (2.5). To estimate solutions to these radial equations in Sect. 2.2, we compare them to some more simple Sturm-Liouville equations. To do this we use the following classical theorems:

Theorem B.1 (Sturm-Picone Comparison Theorem, [7, Theorem B]) Let $y_{1}$ and $y_{2}$ be non-zero solutions to

$\left(p_{1}(x) y_{1}^{\prime}(x)\right)^{\prime}+q_{1}(x) y_{1}(x)=0$,

$\left(p_{2}(x) y_{2}^{\prime}(x)\right)^{\prime}+q_{2}(x) y_{2}(x)=0$,

on the interval $[a, b]$. Assume that $0<p_{2} \leq p_{1}$ and $q_{1} \leq q_{2}$, and let $z_{1}$ and $z_{2}$ be two consecutive zeros of $y_{1}$. Then either $y_{2}$ has a zero in the interval $\left(z_{1}, z_{2}\right)$, or $y_{1}=y_{2}$.

Theorem B.2 (Sturm Comparison Theorem, [6, Chap. 13.7]) Let $y_{1}>0$ on $(a, c)$ and $y_{2}$ be non-zero solutions to $\left(p(x) y_{1}^{\prime}(x)\right)^{\prime}+q_{1}(x) y_{1}(x)=0$,

$\left(p(x) y_{2}^{\prime}(x)\right)^{\prime}+q_{2}(x) y_{2}(x)=0$,

on the interval $(a, c) \subset[a, b]$. Assume that $p>0$ and $q_{2} \leq q_{1}$ on $[a, b]$. Furthermore, assume that

$y_{1}(a)=y_{2}(a) \geq 0 \quad$ and $\quad y_{1}^{\prime}(a)=y_{2}^{\prime}(a) \geq 0$.

Then $y_{1}(x)<y_{2}(x)$ for all $x \in(a, c)$.

It is also important for us to estimate the maximum of some solutions to the Helmholtz equation. To limit the search, we use the following theorem:

Theorem B.3 (Sonin-Pólya Oscillation Theorem, [6, Chap. 13.7]) Let $y$ be a solution of the differential equation

$\left(p(x) y^{\prime}(x)\right)^{\prime}+q(x) y(x)=0$,

where $p$ and $q$ are continuously differentiable functions on $[a, b]$. Suppose that $p>0, q \neq 0$ and $(p q)^{\prime}>0$ on $(a, b)$. Then the successive local maximums of $|y(x)|$ form a decreasing sequence.

Acknowledgements The authors are very grateful to the anonymous referee for useful comments. Their suggestions, in particular, substantially improved the presentation in Sect. 3. The second author was partially supported by NSF Grant DMS-1956294 and by the Research Council of Norway, Project 275113.

Funding Open access funding provided by NTNU Norwegian University of Science and Technology (incl St. Olavs Hospital - Trondheim University Hospital).

Open Access This article is licensed under a Creative Commons Attribution 4.0 International License, which permits use, sharing, adaptation, distribution and reproduction in any medium or format, as long as you give appropriate credit to the original author(s) and the source, provide a link to the Creative Commons licence, and indicate if changes were made. The images or other third party material in this article are included in the article's Creative Commons licence, unless indicated otherwise in a credit line to the material. If material is not included in the article's Creative Commons licence and your intended use is not permitted by statutory regulation or exceeds the permitted use, you will need to obtain permission directly from the copyright holder. To view a copy of this licence, visit http://creativecommons.org/licenses/by/4.0/.

\section{References}

1. Alessandrini, G., Rondi, L., Rosset, E., Vessella, S.: The stability for the Cauchy problem for elliptic equations. Inverse Probl. 25(12), 123004, 47 (2009)

2. Anantharaman, N., Léautaud, M., Macià, F.: Wigner measures and observability for the Schrödinger equation on the disk. Invent. Math. 206(2), 485-599 (2016) 
3. Burman, E., Nechita, M., Oksanen, L.: Unique continuation for the Helmholtz equation using stabilized finite element methods. J. Math. Pures Appl. 9(129), 1-22 (2019)

4. Camporesi, R., Higuchi, A.: Spectral functions and zeta functions in hyperbolic spaces. J. Math. Phys. 35(8), 4217-4246 (1994)

5. Donnelly, H., Fefferman, C.: Nodal sets of eigenfunctions on Riemannian manifolds. Invent. Math. 93(1), 161-183 (1988)

6. Duren, P.: Invitation to Classical Analysis: Pure and Applied Undergraduate Texts, vol. 17. American Mathematical Society, Providence (2012)

7. Hinton, D.: Sturm's 1836 oscillation results evolution of the theory. In: Sturm-Liouville Theory, pp. 1-27. Birkhäuser, Basel (2005)

8. Hrycak, T., Isakov, V.: Increased stability in the continuation of solutions to the Helmholtz equation. Inverse Probl. 20(3), 697-712 (2004)

9. Isakov, V.: Inverse Problems for Partial Differential Equations. Applied Mathematical Sciences, vol 127, 3rd edn. Springer, Cham (2017)

10. Isakov, V., Kindermann, S.: Subspaces of stability in the Cauchy problem for the Helmholtz equation. Methods Appl. Anal. 18(1), 1-29 (2011)

11. Landis, E.M.: A three-spheres theorem. Dokl. Akad. Nauk SSSR 148, 277-279 (1963)

12. Logunov, A.: Nodal sets of Laplace eigenfunctions: polynomial upper estimates of the Hausdorff measure. Ann. Math. (2) 187(1), 221-239 (2018)
13. Logunov, A.: Nodal sets of Laplace eigenfunctions: proof of Nadirashvili's conjecture and of the lower bound in Yau's conjecture. Ann. Math. (2) 187(1), 241-262 (2018)

14. Logunov, A., Malinnikova, E.: Quantitative propagation of smallness for solutions of elliptic equations. In: Proceedings of the International Congress of Mathematicians-Rio de Janeiro 2018. Invited Lectures, vol III, pp 2391-2411. World Scientific Publishing, Hackensack (2018)

15. Malinnikova, E.: The theorem on three spheres for harmonic differential forms. In: Complex Analysis, Operators, and Related Topics. Operator Theory: Advances and Applications, vol 113, pp 213-220. Birkhäuser, Basel (2000)

16. Mangoubi, D.: The effect of curvature on convexity properties of harmonic functions and eigenfunctions. J. Lond. Math. Soc. (2) 87(3), 645-662 (2013)

17. Olver, F.W.J.: Asymptotics and Special Functions. AKP Classics. A K Peters, Ltd., Wellesley (1997). Reprint of the 1974 original [Academic, New York; MR0435697 (55 8655)

18. Petersen, P.: Riemannian Geometry. Graduate Texts in Mathematics, vol 171, 3rd edn. Springer, Cham (2016)

19. Subbarayappa, D.A., Isakov, V.: On increased stability in the continuation of the Helmholtz equation. Inverse Probl. 23(4), 1689-1697 (2007)

Publisher's Note Springer Nature remains neutral with regard to jurisdictional claims in published maps and institutional affiliations. 